\title{
BIKO: TALHER DESCARTÁVEL PARA PEQUENAS PORÇÕES DE ALIMENTOS
}

Claudio Freitas de Magalhães

NEXT PUC-Rio

next.pucrio@gmail.com

Daniel Ruben Nasajon

NEXT PUC-Rio

Resumo: Biko é um projeto desenvolvido durante a pesquisa "Plano das Ideias" que envolve um programa de projetos experimentais voltados para questionar o caráter analítico/sintético das metodologias de projeto de produtos. O objetivo é inverter este carácter partindo de explorações com as estruturas físicas e materiais para posteriores definições de produtos. Para viabilizar explorações físicas diretas foram privilegiadas explorações com materiais disponíveis em chapas planas assim como foram utilizadas diversas tecnologias de fabricação digital. A partir de explorações iniciais sistemáticas e não sistemáticas com cortes e vincos em um papel especial foi desenvolvido um talher em forma de pinça que pode ser usado para alcançar pequenas porções de alimentos (finger foods) como: catering de eventos, comida asiática, queijos e vinhos, petiscos, churrascos, etc. O produto criado é simples, higiênico, reciclável, versátil, econômico, fácil de estocar e pode substituir o uso de guardanapos, espetos, garfos, hashis (talher asiático), dentre outros. Após a entrada de pedido de registro no INPI, o projeto foi exposto no Rio+Design 2015 no Jockey Club do Rio de Janeiro e também na Semana de Design de Milão 2016, no stand do Rio+Design na via Tortona. Foi o primeiro colocado no prêmio "Meu Futuro Negócio" de 2015 organizado pelo IEL (Instituto Euvaldo Lodi) em parceria com a incubadora de empresas, Instituto Gênesis, sendo premiado com um período de incubação na instituição.

Palavras-chave: metodologia de design, design exploratório, alimentação, design de produto

\begin{abstract}
Biko is a project developed during the "Ideas Plan" research which involves a program of experimental projects aimed to question the analytical/synthetic characteristics of product design methodologies. The goal is to reverse this character, starting from physical structures and materials exploration before future product definitions. Available materials in flat sheets as well as several digital manufacturing technologies were privileged to enable direct physical exploration. From systematic and nonsystematic initial explorations with cuts and creases on a special paper, it was developed a piece of flatware like pincers that can be used to reach for
\end{abstract}


small portions of food (finger foods) such as for catering events, Asian food, cheeses and wine, snacks, barbecues, etc. The developed product is simple, hygienic, recyclable, versatile, economical, easy to store and can replace the use of napkins, skewers, forks, chopsticks (Asian cutlery), among others. After the patent pending registration request at INPI (National Institute of Industrial Property), the project was exposed at Rio+Design showroom in 2015 in the Jockey Club in Rio de Janeiro and also in Milan Design Week 2016 at the Rio+Design stand in via Tortona. The project got first placed on the "My Future Business" competition in 2015, organized by IEL (Euvaldo Lodi Institute) in partnership with the business incubator, Genesis Institute, being awarded an incubation period in the institution.

Keywords: design methodology; exploratory design; alimentation; product design

\section{INTRODUÇÃO}

A maior parte dos produtos vem evoluindo de forma incremental a partir de um processo de design convencional (PUGH, 1990). A configuração de um carro é a mesma desde que surgiu o conceito de carro. Um dos aspectos deste processo é a divisão entre o espaço do problema e o espaço da solução. Pode ser dito que esta divisão problema-solução caracterizaria um projeto, ou seja, a solução surge de um problema pré-definido e anteriormente analisado. A eficiência desta sequencialidade é questionada pela velocidade das mudanças. Para resolver esta questão, propõe-se uma maior sobreposição das etapas do processo de projeto como meio para reduzir tempo e recursos. Desta forma, contextos dinâmicos ou produtos inovadores exigiriam processos com maior sobreposição entre o espaço do problema e da solução em um projeto (IANSIT, 1995). Em uma situação extrema, em projetos altamente inovadores, ligados a estratégias de previsão do futuro, esta sequência pode ser invertida. Partindo de experimentações, algumas empresas de ponta questionam suas estratégias, redirecionam conhecimentos e capacitações tecnológicas a partir concepções de produtos (VISION OF THE FUTURE, 1996). Desassociam o projeto de um problema específico pré-determinado, porém, relacionado com algum aspecto que caracteriza seu ambiente, seus recursos, novas tecnologias ou com seu contexto futuro de atuação. Observa-se assim, a contextualização do método ao projeto (BAXTER, 1995, PMI, 1996). A fase da análise do problema, ou melhor, a fase inicial do pré-projeto ou fuzzy front end é a chave para conseguir um descolamento de uma estrutura de produto já existente, ou seja, de concepções institucionalizadas. No entanto, a maioria das empresas encontra muitas justificativas para manter suas estratégias e processos de desenvolvimento dentro de parâmetros conhecidos e muitas vezes também utilizando processos reativos, até mesmo optando pela cópia como estratégia de desenvolvimento de produtos para evitar riscos inerentes da inovação.

Por outro lado, a partir do início doa anos 90, observamos o crescimento do interesse na área do Design sobre os aspectos que antecedem a concepção de produtos, definidos como os conteúdos concernentes a um Design Estratégico (MAGALHÃES, 1994). Tanto o exercício profissional quanto o ensino e a pesquisa do design passaram demonstrar interesse sobre este tema e a incorporar discursos e 
práticas voltadas principalmente para a fase anterior à definição do projeto e do produto a ser desenvolvido. Nesta fase, de carácter principalmente analítico se busca um entendimento da situação de projeto e uma maior fundamentação da definição do produto. Utiliza-se para isso uma série de métodos para análises do ambiente de negócios, mercadológico, tecnológico, social e cultural, oriundas de diversas áreas do conhecimento. O Design Estratégico absorveu inicialmente a cultura do Marketing e da Gestão da Qualidade. As consultorias de design no Brasil foram influenciadas pelo contato com as agencias de publicidade que terceirizavam a maioria dos projetos que não fossem anúncios e publicidade. E o Branding, que foi a base teórica de sustentação do discurso estratégico do design e permanece até o momento, também teve sua origem na área da administração de marcas. Assim, o Design Estratégico foi se estabelecendo. $O$ discurso estratégico do design de algum modo foi sendo incorporado ao do universo da administração, em especial através do "Design Thinking" (CROSS, 2001) e sendo divulgado por Tim Brown (2009) e seu estúdio IDEO (http://www.ideo.com ). Portanto, se verifica que o Design Estratégico, não pouco pertinente e necessário em um primeiro momento, representa um dos paradigmas preponderantes no ensino e principalmente na pratica profissional nos dias atuais. Desta maneira, se constata uma ênfase no processo analítico e um desequilíbrio entres fundamentos do design, especialmente em detrimento do conhecimento e vivência dos elementos básicos da forma e da composição. Ou seja, estratégias centradas no núcleo da concepção de produtos, oriundas de uma experimentação na ação, são restritas a uma fase de síntese, delineada por uma fase analítica do projeto, e por um briefing de produto que não contempla investigações no campo da materialidade ou da configuração. Esta situação é determinada pela utilização de uma estrutura específica do método de design, onde se determina uma rígida distinção entre uma fase analítica anterior e uma fase de síntese posterior.

\section{DESENVOLVIMENTO}

Este projeto pretendeu investigar a potencialidade de inovação da geração de conceitos e soluções, a partir da exploração da transformação do plano, antecedendo a definição de problemas e ou oportunidades de projeto, utilizando transposições entre mídias físicas e digitais para a realização de modelos e protótipos experimentais. Para efeito da delimitação desta pesquisa, pretendeu-se estudar o contexto dos produtos produzidos a partir de materiais transformados e disponibilizados em chapas planas, como papéis, chapas metálicas e plásticas. Pretendeu-se partir da solução para o problema: através da geração de protótipos e soluções formais genéricas chega-se ao problema de projeto. A situação de projeto é induzida pela solução.

A metodologia tradicional de Design se adequa ao desenvolvimento incremental do produto, partindo de modo geral de uma problematização para posterior construção de soluções direcionadas ao resultado desta primeira análise do problema. Desta maneira, são analisadas uma série de aspectos para que depois de uma síntese integrada de fatores, funções e parâmetros se siga para a concepção, que envolve dar uma forma integrada a estes elementos. Na metodologia aplicada à pesquisa "Plano das Ideias" as fases de desenvolvimento de produtos são invertidas, colocando a concepção e a análise da forma e dos materiais como diretriz para novos princípios de produtos. 
A partir de princípios desenvolvidos e explorados durante o início da pesquisa, alguns foram selecionados e melhor desenvolvidos para a geração de aplicações do mesmo em novos produtos. Um processo menos estruturado com potencial de inovação no desenvolvimento de produtos.

Após uma primeira análise experimental voltamos o projeto para a indústria alimentícia, desenvolvendo então um talher para finger food. O produto foi denominado de Biko por causa de sua forma, semelhante à um bico de pássaro. Desta forma, iniciamos o processo de detalhamento técnico que, por fim, alinharia as necessidades do mercado aos requisitos sanitários e as questões de sustentabilidade.

Com o projeto definido e devidamente protegido com o depósito de patente, um modelo de negócios foi desenvolvido para a inserção do produto no mercado. 0 projeto-foi primeiro colocado no concurso "Meu Futuro Negócio" em 2015. Foi exposto no Rio+Design 2015 no Jockey Club do Rio de Janeiro e posteriormente na Semana de Design de Milão em Abril de 2016.

\subsection{Metodologia "Plano das Ideias"}

Visando identificar a potencialidade da geração de formas a partir da transformação do plano como incitação para soluções ou desenvolvimento de produtos inovadores, a metodologia empregada reviu estudos e tratados sobre princípios e fundamentos da forma e da configuração (HANNAH, 2002, DONDIS, 1991, GOMES FILHO, 2000; WONG, 1993; ), técnicas de criatividade (BAXTER, 1995) e estudos específicos (ROCHA, 2000; YIGIT, 2004; VYZOVITI, 2006). Esta revisão de literatura apoiou a geração de formas a partir da transformação do plano. Para esta transformação do plano foram utilizados recursos e simulações físicos e digitais, como desenho 2D e 3D estáticos, assim como transformação dinâmica da forma em meio físico e digital, através de modelagem 3D tradicional e modelagem paramétrica. $O$ registro sistemático através de fotos e pequenas filmagens, seguidas de análise da configuração estética e classificação desta geração servem como reflexão sobre o processo criativo e para decisões de aperfeiçoamentos e mudanças necessárias, assim como, para a exploração concentrada nos modelos promissores. Foram realizadas comparações entre os diferentes processos e resultados nas mídias físicas e digitais. Foram realizadas associações dos modelos tridimensionais gerados com oportunidades de projeto de produtos. Foram aplicadas técnicas de Modelo Simples em escala reduzida (LUCCI e ORLANDINI, 1989), assim como protótipos em escala natural. Ambos os modelos e protótipos foram produzidos através de técnicas tradicionais ou por impressão 3D. Foi realizada pesquisa iconográfica de produtos desenvolvidos com transformações de planos feitos de diversos materiais. A partir de abertura de oportunidades e princípios funcionais foram realizados experimentos voltados para aplicações e usos.

\subsection{Primeiros estudos}

Os primeiros estudos foram feitos a partir de cortes e vincos em espuma EVA. Dezenas de experimentos foram desenvolvidos dando origem a princípios de dobra e comportamentos diversos no material escolhido. 


\subsection{Exploração de princípio}

A partir dos estudos feitos diversos princípios foram notados. Ainda sem nenhum tipo de predefinição de algum problema ou aplicação um princípio foi selecionado (Figura 1) para, desta vez, a ser explorado com mudanças de escala, material e processo (Figuras 2, 3, 4 e 5). Desta forma, esperava-se abranger as possibilidades de inovação e aumentar a capacidade de associação para os produtos a serem desenvolvidos posteriormente.

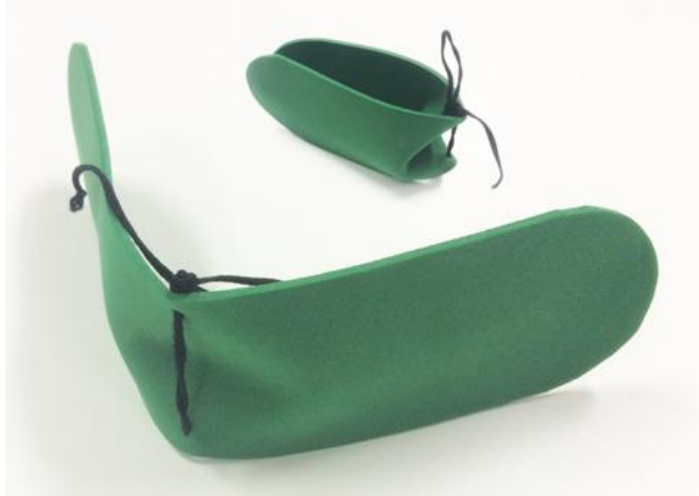

Figura 1 - Primeiro modelo feito com o princípio de dobra central

Fonte: Elaborado pelo autor, com base na pesquisa realizada

\section{Figura 2 - Mudança de material e escala (PP)}

Fonte: Elaborado pelo autor, com base na pesquisa realizada
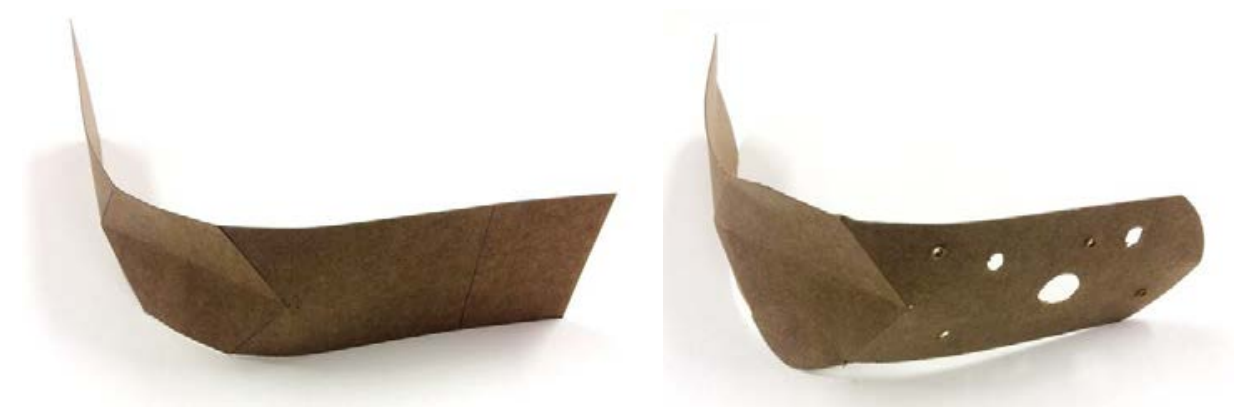

Figura 3 e 4 - Mudança de material e escala (Papel Kraft)

Fonte: Elaborado pelo autor, com base na pesquisa realizada 


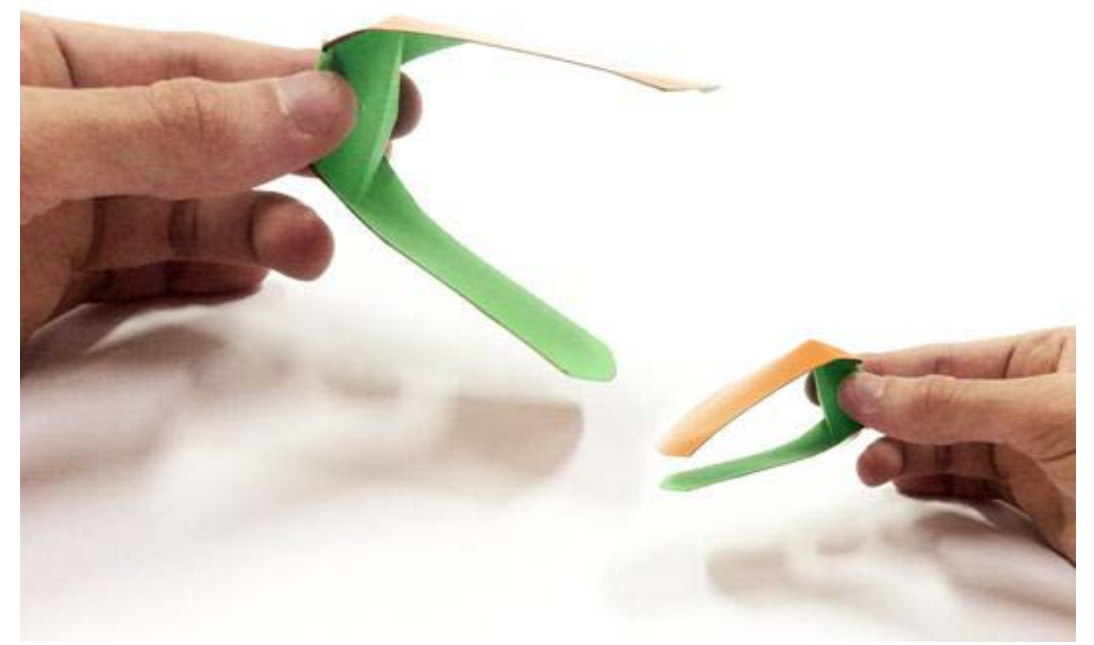

Figura 5 - Mudança de material e escala (Papel e Vinil)

Fonte: Elaborado pelo autor, com base na pesquisa realizada

\subsection{Definição de produto}

Neste estágio foram aplicadas funções para as formas desenvolvidas a partir do princípio selecionado. Do último sketch tridimensional construído (Figura 5) foi idealizado um talher para finger food, ou seja, alimentos em pequenas porções que podem ser manipuladas com os dedos. Alguns dos exemplos de uso estão na culinária asiática, catering de festas, churrascos, etc.

A partir desta forma seria necessário detalhar o projeto levando em consideração o seu contexto de uso, concorrentes, processos de fabricação, distribuição, etc. Sua forma, material e processo deveriam ser estabelecidos levando em consideração a proposta de uso do projeto. Mais de 100 modelos foram desenvolvidos até a definição da forma, material e processo mais promissores. As figuras 6 a 14, mostram alguns dos modelos desenvolvidos com o intuito de explorar os cortes e vincos em diferentes materiais.

Figura 6 e 7 - Variação de forma e de material (PP)

Fonte: Elaborado pelo autor, com base na pesquisa realizada

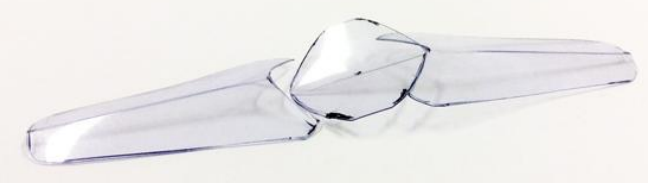

Figura 8 - Variação de forma e material (PET)

Fonte: Elaborado pelo autor, com base na pesquisa realizada 


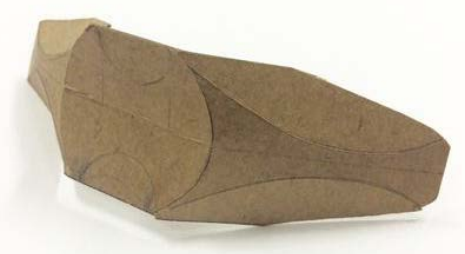

Figura 9 - Variação de forma e material (papel kraft)

Fonte: Elaborado pelo autor, com base na pesquisa realizada
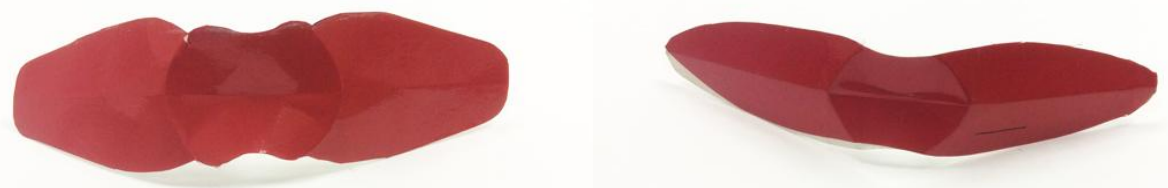

Figura 10 e 11 - Variação de forma e material (triplex com vinil)

Fonte: Elaborado pelo autor, com base na pesquisa realizada

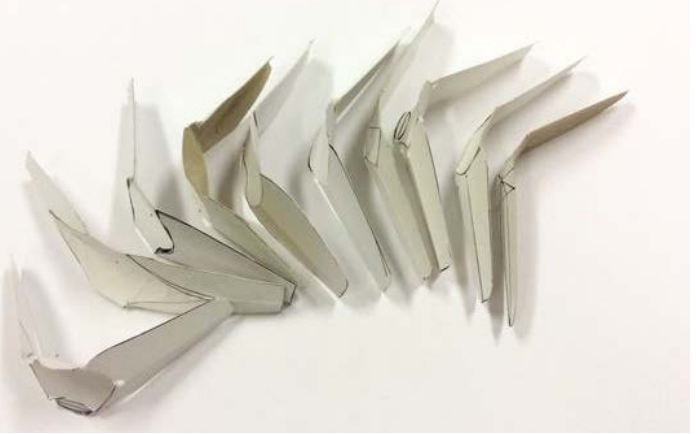

Figura 12 - Algumas modelos com variações de corte e vinco em papel Triplex Fonte: Elaborado pelo autor, com base na pesquisa realizada
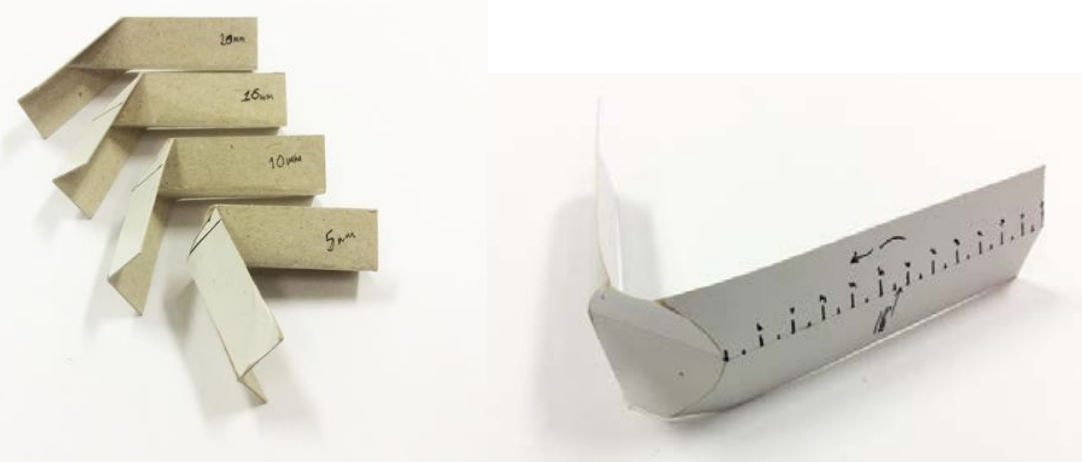

Figura 13 e 14 - Estudos sobre o trabalho do papel em relação ao ângulo do vinco Fonte: Elaborado pelo autor, com base na pesquisa realizada

Ao final da concepção, o produto foi definido com sendo um talher descartável para alimentação informal com as mãos em eventos ou tipo "finger foods", devendo oferecer as seguintes funções:

- Comunicar seu funcionamento inovador aos novos usuários;

- Transferir pressão suficiente da base para as pontas para pegar pequenas quantidades de alimentos;

- Ter estrutura durante a pressão de modo que as pontas não dobrem ao pegar os alimentos; 
- Não machucar os dedos ou a boca dos usuários;

- Resistir ao contato com alimentos úmidos;

- Permitir a possibilidade de impressão;

- Ser adequado ao descarte sem agressão ao meio ambiente.

\subsection{Detalhamento técnico}

Uma vez que o produto seria usado manualmente e colocado em contato com alimentos, o projeto necessitava estabelecer alguns parâmetros a serem alcançados para seu bom funcionamento como a questão de higiene e adequação às normas da área.

Considerando principalmente o manuseio, a aplicação da força e o comportamento funcional e dos materiais foram realizados diferentes testes com usuários com protótipos e cabeças de série produzidos com o processo de corte e vinco.

\subsubsection{Capacidade de Impressão}

A possibilidade de impressão é uma característica que diferencia o produto de seus concorrentes diretos. Uma vez que o teste com papel comum fora testado em alimentos mais gordurosos e líquidos sem resultados positivos, optamos pela utilização de um papel sintético a base de polipropileno próprio para alimentos que aceita impressão.

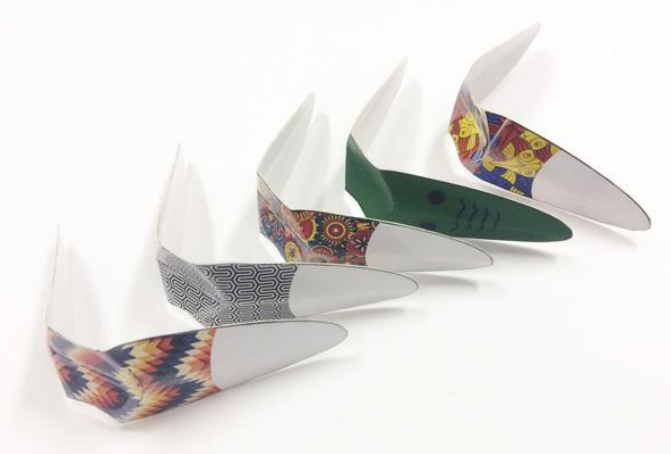

Figura 15 - Modelos impressos em papel triplex e plastificação

Fonte: Elaborado pelo autor, com base na pesquisa realizada

\subsubsection{Produção}

O processo de corte e vinco foi selecionado para os primeiros testes de produção devido a sua escala e custos. Foram feitas facas gráficas sob encomenda para a continuidade de testes sobre um novos materiais ainda mais resistentes.
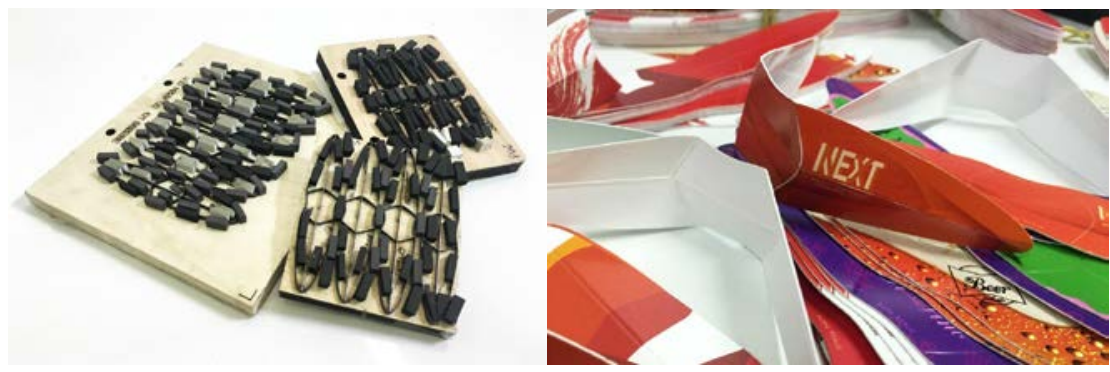

Figura 16 e 17 - Facas gráficas para corte e vinco com diferentes tipos de acabamento e produtos finalizados em YUPO (PP), respectivamente

Fonte: Elaborado pelo autor, com base na pesquisa realizada 


\subsubsection{Sustentabilidade}

O Biko é um talher $100 \%$ reciclável e atóxico. Relacionando o projeto a um hashi (talher asiático) descartável de madeira, o Biko evitaria a derrubada de cerca de 4 milhões de árvores anualmente, apenas na china (Greenpeace).

\subsection{Proteção Legal}

Para que o produto fosse exposto ao público sem problemas legais futuros, uma patente e 3 desenhos industriais foram preparados e depositados junto ao INPI. A proteção foi feita junta ao departamento legal da AGI (Agência de Inovação da universidade) e encontra-se agora em situação de patente pendente. A proteção inclui parâmetros que não limitam a forma externa do dispositivo, mas que abrangem para uma variedade ilimitada de contornos diferentes.

\subsection{Finalização do produto}

O produto foi finalizado medindo $160 \mathrm{~mm}$ de comprimento e $30 \mathrm{~mm}$ de largura na forma plana e possui aproximadamente $0.5 \mathrm{~mm}$ de espessura. É produzido em YUPO, um papel sintético a base de polipropileno que é ao mesmo tempo atóxico, impermeável e resistente a rasgos.
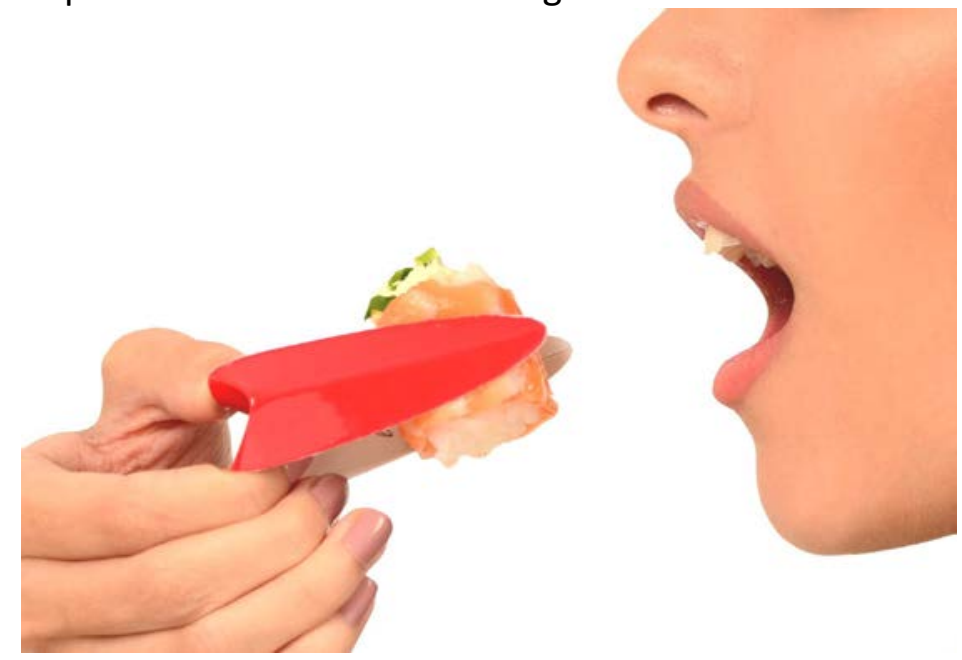

Figura 18 - Biko sendo usado para pinçar sushi

Fonte: Elaborado pelo autor, com base na pesquisa realizada

\subsection{Modelo de negócios}

O Biko possui uma ampla versatilidade de aplicações no mercado. Além de servir para reduzir custos de restaurantes, o Biko ainda é uma plataforma de merchandising já que possui $100 \%$ da superfície de contato com espaço para impressão. Assim, o talher pode ser aplicado em fins decorativos ou publicitários. Foram desenvolvidos 3 modelos de negócio atrelados ao projeto como mostra a Figura 19. 


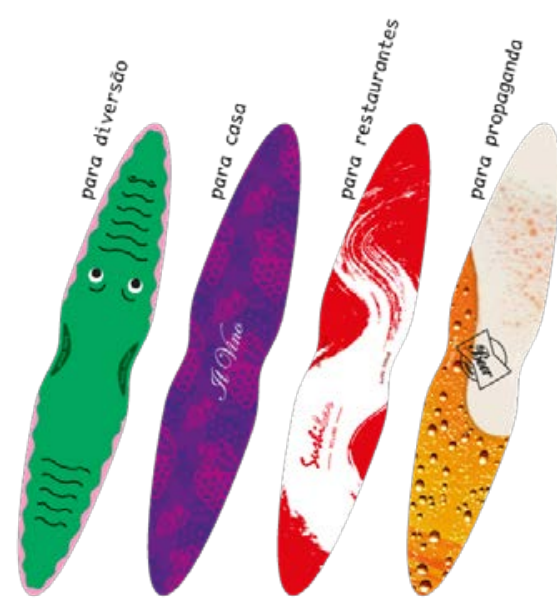

Figura 19 - Aplicação de estampas diferentes para cada modelo de negócios

Fonte: Elaborado pelo autor, com base na pesquisa realizada

\subsubsection{Para Pessoa Física}

O projeto prevê a possibilidade de comprar Bikos com estampas padronizadas (como mostra as duas primeiras estampas da Figura 19) em pacotes de 100 unidade para serem usados em eventos como churrascos, queijos e vinhos etc.

\subsubsection{Para Pessoa Jurídica}

O projeto prevê a possibilidade de ser vendido em grandes quantidades com estampas personalizadas (como mostra a terceira estampa da Figura 19) para empresas que querem expor a sua marca nesta plataforma.

\subsubsection{Para Subsídio}

Uma empresa que queira usar a plataforma de merchandising como uma estratégia publicitária pode subsidiar Bikos, fabricados em grande volumes, para terceiros (como mostra a quarta estampa da Figura 19).

\subsection{Eventos e Prêmios}

O produto foi primeiramente exposto no Rio+Design 2015, organizado pelo 0 Globo no Jockey Club do Rio de Janeiro. Milhares de visitantes tiveram um contato direto com o produto e obtivemos um retorno extremamente positivo da opinião do público.

No final de 2015, o projeto foi o primeiro colocado no prêmio "Meu Futuro Negócio" organizado pelo IEL (Instituto Euvaldo Lodi) em parceria com a incubadora de empresas Instituto Gênesis. O projeto foi contemplado com 6 meses de incubação no Instituro Gênesis que se iniciou em fevereiro de 2016.

Em Abril de 2016 foi convidado a expor no Rio+Design Milão, que ocorreu na zona Tortona durante a Semana de Design de Milão, um dos maiores eventos de Design no mundo.

\section{CONCLUSÃO}

O projeto derivado de um desenvolvimento de produtos não tradicional, não focado no cliente ou no mercado se apresenta com resultados positivos até o momento com relação a estes aspecto. O produto final demonstra a virtude da 
metodologia aplicada na pesquisa "Plano das Ideias" para a criação de novos produtos inovadores que possivelmente não seriam gerados tomando partida de um processo de design tradicional. Uma vez que o processo de projeto não é previamente contagiado com referências externas de contexto e problemática, um projeto pode ser desenvolvido de forma mais livre, a partir de estudos de princípios da forma, dando origem a novas possibilidades de projetos e ideias inovadoras. Cabe ressaltar que este tipo de projeto experimental deve ter o seu lugar nas estratégias de desenvolvimento de produtos. Em especial, o caráter exploratório tem grande probabilidade de riscos e ao erro quando se tem uma meta ou propósito predeterminado, como na maioria dos processos de design. Desta maneira, este tipo de processo pode ser adequado à uma estratégia exploratória que antecede ou é paralela e independente dos processos tradicionais focados em um mercado-produto predefinidos, incluindo assim, recursos

financeiros a fundo perdido, entendidos como uma das possibilidades de investimentos em inovação pelo design.

\section{REFERÊNCIAS}

BAXTER, Mike. Projeto de Produto - Guia prático para o desenvolvimento de novos produtos. 1.ed. São Paulo: Edgarg Blücher Editora, 1998.

BROWN, T. Change by Design: how design thinking can transform organizations and inspire innovation. NewYork: Harper Business, 2009.

CROSS, Nigel. Designerly Ways of Knowing: Design Discipline Versus Design Science Design Issues: Volume 17, Number 3 Summer 2001.

DONDIS, A. D., A Primer of Visual Literacy. Cambridge: MIT Press, 1973 (ed. bras. A

Sintaxe da Linguagem Visual, São Paulo, Martins Fontes, 1991).

GOMES FILHO, João. Gestalt do Objeto: Sistema de leitura visual da forma. 1.ed. São Paulo: Escrituras Editora, 2000.

GREENPEACE. Turning chopsticks into trees, 2010. Disponível em: <http://www.greenpeace.org/eastasia/news/stories/forests/2010/chopsticks-trees/>. Acesso em: 21 de mai. 2016

HANNAH, G. G. Elements of Design: Rowena Red Kostellow and the structure of visual relationships. Princeton Architectural Press, New York, 2002.

IANSITI, Marco. Shooting the Rapids: Managing Product Development in Turbulent Environments. California Management Review, Vol. 38, No. 1 Fall, 1995.

LUCCI, R. e ORLANDINI, P., Product Design Models, Van Nostrand Reinhold, New York, 1989.

MAGALHÃES, Claudio F., Formas do Design: por uma metodologia interdisciplinar. 2.ed. Rio de Janeiro: Rio Book's, 2014.

MAGALHÃES, Claudio F., Design Estratégico: integração e ação do Design Industrial dentro das empresas. Rio de Janeiro, SENAI/DN, SENAI/SETIQT, CNPq, IBICT, PADCT, TIB, 1997.

PUGH, Stuart. Total Design - Integrated Methods for Successful Product Engineering. 3.ed. UK, Addison-Wesley, Wokingham, 1990. 
ROCHA, Carlos Sousa. Plasticidade do Papel e Design. Lisboa: Plátano Editora, 2000.

Vision of The Future, Philips Corporate Design, Eindhoven, The Netherlands (1996)

VYZOVITI, Sophia, Supersurfaces: Folding as a method of generating forms for architecture, products and fashion. BIS Publishers, Amsterdam, Holanda, 2006.

WONG, Wucius. Princípios de Forma e Desenho. São Paulo: Martins Fontes, 1998.

YiĞiT, Nergiz. Industrial Product Design by Using Two-Dimensional Material in the Context of Origamic Structure and Integrity. İzmir, Turquia, 2004. Dissertação

(mestrado em Desenho Industrial) - Faculdade de Desenho Industrial, İzmir Institute of Technology. 